\title{
Correlating Rheological Experiments, TEVP Models, and Microstructure with Small Angle Light Scattering
}

\author{
Matthew J. Armstrong*, André M. Pincot \\ Department of Chemistry and Life Science, United States Military Academy, West Point, NY, USA \\ Email: *matthew.armstrong@westpoint.edu
}

How to cite this paper: Armstrong, M.J. and Pincot, A.M. (2022) Correlating Rheological Experiments, TEVP Models, and Microstructure with Small Angle Light Scattering. Open Journal of Fluid Dynamics, 12, 36-55.

https://doi.org/10.4236/ojfd.2022.121002

Received: November 29, 2021

Accepted: February 13, 2022

Published: February 16, 2022

Copyright $\odot 2022$ by author(s) and Scientific Research Publishing Inc. This work is licensed under the Creative Commons Attribution International License (CC BY 4.0).

http://creativecommons.org/licenses/by/4.0/

\begin{abstract}
The existence of an evolving microstructure in a 2.9 vol\% fumed silica in paraffin oil and polyisobutylene is demonstrated experimentally and via rheological modeling during steady state and large amplitude oscillatory shear flow. The continuously evolving, rebuilding, and breaking down of the microstructure is shown, and correlated through the rheology experiments, thixo-elastovisco-plastic modeling, and small angle light scattering (SALS). All elements are then connected via a global, stochastic optimization algorithm that will provide parameter estimation with a "best-fit" of the steady state and transient data using the well-known Modified Delaware Thixotropic Model, allowing for the comparison of SALS results with experimental values.
\end{abstract}

\section{Keywords}

Small Angle Light Scattering, Microstructure, Rheology,

Thixo-Elastic-Viscoplastic Materials

\section{Introduction}

Here we demonstrate the use of the full soft matter interrogation technique, consisting of simultaneous use of rheological and scattering data along with modeling, and parameter estimation to interrogate a complex material's microstructure. The model incorporated here is the recently published Modified Delaware Thixotropic Model (MDTM) [1] [2] [3]. The fitting of the model parameters to the steady state rheological data is accomplished with a novel parallel tempering algorithm [1] [2] [4]. Steady state and large amplitude oscillatory shear (LAOS) scattering and rheological data from the TA Instruments DHR-3 stress-controlled rheometer is wielded to make structural connections between 
the MDTM predictions of structure, $\lambda$, with measures of shear induced structure from the scattering analysis. In addition to this, Marty's number, $M^{\prime}$ (akin to an inverse Bingham number), and viscosity measurements from steady state and LAOS are used to facilitate discussion of key structure development and evolution under flow. Our goal is to seek evidence correlating the alignment factor, $A_{f}$ and $\lambda$ under flow. Furthermore, the scattering measurement methods are discussed, and it is demonstrated that it can be used to calculate structural features under varying flow conditions. Discussion of neutron scattering experiments for our model thixotropic material, 2.9 vol\% fumed silica in paraffin oil and polyisobutylene, are included. Following this, the results of the scattering experiments using small angle light scattering for the same system are shown and discussed. Lastly, the scattering, modeling, and rheology data is interpreted [1] [5] [6] [7].

Much work has been done recently combining scattering and rheology to develop structure-property relations for the non-Newtonian flow of complex fluids [1] [8] [9] [10] [11]. Scattering in general is used to gain additional knowledge of microstructural evolution during rheological experiments of varying shear rate. Small angle neutron scattering (SANS) has been incorporated several recent research groups [10]-[22]. Small angle light scattering has also been a frequently used method to gain insight about microstructure, particularly at smaller scales than SANS [23] [24]. There are two goals achieved under flow rheological experiments: to determine microstructural rearrangement during flow through the intensity $(I(q))$ versus $q$ (inverse length scale) curve; and determine flow-induced anisotropy via the $I(q)$ vs. $q$ information. The alignment factor, $A_{f}$ represents the degree of anisotropy in the scattered intensity calculated over a specific $q$ range. Here, $A_{f}$ is a measure of the aggregate alignment about $\phi_{0}$, and the typical values are 0 for the completely isotropic state, to 0.7 for a completely "anisotropic" state [1] [6]. Thus, our first goal is to probe these metrics of scattering from a model thixotropic material under various combinations of frequency and strain amplitude under LAOS conditions, with scattering, and use the scattering data to calculate $A_{f}$.

We follow this with a theoretical discussion in Section 2 consisting of the MDTM TEVP model description, small angle neutron scattering and small angle light scattering. This is followed by the Materials and Methods section consisting of experimental methods and model parameter fitting procedures. Following this we present Results and Discussion section, and end with Conclusions.

\section{Theoretical Section}

\subsection{MDTM Description}

Having calculated the alignment factor using the $I(q) \vee q$ data, a recent scalar, thixotropic model, the Modified Delaware Thixotropic Model (MDTM), is applied to make predictions for structure, $\lambda$, and make comparisons to look for any correlations between the degree of structural alignment caused by flow, and the lambda prediction using the MDTM. Like other recent thixotropic peer models 
[25] this model includes an additional, separate timescale for $\lambda$ :

$$
\begin{gathered}
\frac{\mathrm{d} \lambda}{\mathrm{d} t}=k_{\text {Brown }}\left[-\lambda\left|\hat{t}_{r 1} \gamma_{p}\right|^{a}+(1-\lambda)\left(1+\left|\hat{t}_{r 2} \gamma_{p}\right|^{d}\right)\right], \\
\hat{t}_{r 1}=\left(\frac{k_{\text {Break }}}{k_{\text {Brown }}}\right)^{\frac{1}{a}} \text {, and } \\
\hat{t}_{r 2}=\left(\frac{k_{\text {Aggr. }}}{k_{\text {Brown }}}\right)^{\frac{1}{d}}
\end{gathered}
$$

where $k_{\text {Brown }}$ is the kinetic constant representing system structure restoration and Brownian activity, while $t_{r 1}$ and $t_{r 2}$ are normalized, thixotropic constants related to the breaking and aggregation of microstructure constants $k_{\text {Break }}$ and $k_{\text {Aggr. }}$. Unlike the original thixotropic model of Dullaert and Mewis (2006) [20] [26], this effort allows for the use of $a$ and $d$ as variable exponents, unlike the original, explicit values of 1 and 0.5 respectively. However, like the original model, the MDTM incorporate a framework that separates strain and shear rate into their constituent elastic and plastic components to enhance the elasto-viscoplasticity demonstrated by some soft solids [1] [2] [26]. This concept originated with the principles of the kinematic hardening of plasticity [27] [28], which dictates that the totality of the strain and shear rates can be described by the addition of their elastic and viscous components:

$$
\gamma_{e}+\gamma_{p}=\gamma \leftrightarrow \dot{\gamma}_{e}+\dot{\gamma}_{p}=\dot{\gamma}
$$

Moreover, the evolution of the elastic strain [29] can be described as such:

$$
\dot{\gamma}_{e}=\dot{\gamma}_{p}-\frac{\gamma_{e}}{\gamma_{\max }}\left|\dot{\gamma}_{p}\right| \text {. }
$$

This addition entails an additional equation for the MDTM modulus:

$$
\frac{\mathrm{d} G_{f}}{\mathrm{~d} t}=-k_{G}\left(G_{f}-\lambda G_{0}\right) .
$$

This term acts to introduce an additional time constant $\propto k_{G}^{-1}$ to the system. Of additional note is form of the strain term, which reflects the model's linear superposition of elastic strain and plastic strain [1]. Analogously, the shear rate is represented by a linear superposition of the system elastic shear rate and plastic shear rate, a similarity demonstrated via Equation (4) above. The plastic component of shear rate is described as:

$$
\dot{\gamma}_{p}= \begin{cases}\frac{\dot{\gamma}}{2-\frac{\left|\gamma_{e}\right|}{\gamma_{\max }}} & \dot{\gamma}>0 \\ \frac{\dot{\gamma}}{2+\frac{\left|\gamma_{e}\right|}{\gamma_{\max }}} & \dot{\gamma}<0\end{cases}
$$

being a function of $\dot{\gamma}, \gamma_{e}$, and $\gamma_{\max }$, respectively representing the shear rate, elastic strain, and maximum strain. $\gamma_{\max }$ specifically represents the maximum 
elastic strain a given material can tolerate and is characterized by the given equation

$$
\gamma_{\max }(\lambda)=\min \left(\frac{\gamma_{C O}}{\lambda^{m}}, \gamma_{\infty}\right) .
$$

where it exists as a function of the structure parameters. Nonetheless, this produces the necessary connective relationship between the yield stress, $\sigma_{y 0}$, and zero deformations strain, $\gamma_{C O}$, to fix the following expression [1]:

$$
\gamma_{C O}=\frac{\sigma_{y 0}}{G_{0}} .
$$

In having detailed the material properties and behaviors encompassed within the updated MDTM, the constitutive equation can be assembled as a linear superposition of the system's elastic and plastic components, where the elastic portion is relevant to reversible, temporary elongation processes and the plastic portion irreversible, permanent dissipative processes. With an additional viscous stress term, the MDTM constitutive equation represents the structural viscosity of the process material via the following equation:

$$
\sigma=G_{f} \gamma_{e}+\lambda K_{S T} \dot{\gamma}_{p}^{n_{2}}+K_{\infty} \dot{\gamma}_{p}^{n_{1}}
$$

where the first term, $G_{f} \gamma_{e}$, represents elastic stress, and the subsequent terms, $\lambda K_{S T} \dot{\gamma}_{p}^{n_{2}}$ and $K_{\infty} \dot{\gamma}_{p}^{n_{1}}$ respectively represent the structural and solvent components of viscous stress with consistency parameters $K_{S T}$ and $K_{\infty}$ and power law parameters $n_{2}$ and $n_{1}$. In application of the MDTM within this effort, $\gamma_{\infty}$ is set to 1 . However, our experimentation revealed no situation in which $\lambda$ attained a sufficiently small value to meet this maximum elastic strain value. This parameter can be dropped to simplify the model. This simplification does not have noticeable effect on subsequent application to the analysis of the experimental material.

Therefore, one hypothesis is that $\lambda$ from the MDTM will correlate with one or both structural features determined from the scattering under flow. Here, the 2.9 vol\% fumed silica in paraffin oil and polyisobutylene dispersion studied in [2], is probed with the neutron and light scattering. In addition to changes in the overall scattering intensity, the alignment factor, $A_{f}$ shown here is calculated [6]

$$
\left|A_{f}\right|(q)=\frac{\int_{0}^{2 \pi} I(q, \theta) \cos \left[2\left(\theta-\theta_{0}\right)\right] \mathrm{d} \theta}{\int_{0}^{2 \pi} I(q, \theta) \mathrm{d} \theta},
$$

where $I(q, \phi)$ am the scattering intensity, $q$ is the scattering vector and $\theta$ is the azimuthal angle. This follows the strategy employed by [8] [13] [15] who explored correlations between these two measures of structure, and the measured rheology.

Additionally, the calculation of the Marty's number can be applied to measure and gauge a "dimensionless", stress value that can fluidize the gel, like an inverse 
Bingham number, and is shown here

$$
M^{\prime}=c \frac{\mu_{s} \dot{\gamma} \varphi^{2}}{\sigma_{y 0}}
$$

where $c$ is a proportionality constant, estimated experimentally, $\mu_{s}$ is the solvent viscosity, $\varphi$ is the particle volume fraction, and $\sigma_{y 0}$ is the yield stress. The $M^{\prime}$ provides a dimensionless shear rate for comparing different materials and has been demonstrated to correlate with both viscosity and microstructure for this sample [7]. The success of this comparison motivates further exploration of our model thixotropic suspensions.

\subsection{Small Angle Neutron Scattering}

The neutron scattering experiments were conducted on the NGB-30 line, at NCNR, NIST, in Gaithersburg, MD, at a series of steady state conditions using the Anton Parr, MCR-300 stress-controlled rheometer as was done in previous work by Lopez-Baron et al. [9], Gurnon et al. [8] [16] and Eberle and Porcar [14]. Figure 1 shows the flow curve as images of the scattering intensity from the 1 - 3 plane. The 2-dimensional scattering patterns at the $13.5 \mathrm{~m}$ detector positions are presented, along with the difference pattern of each with the quiescent pattern. An $I(q)$ vs $q$ curve from two very different shear rates (shown in figure) is also shown, where no changes in microstructure are at these shear rate conditions [1]. Thus, SANS is not able to discern the length scale of our fumed silica aggregates, showing, that even though our fumed silica particles are said to be of diameter $16 \mathrm{~nm}$, the smallest fused fumed silica aggregate is not of this length scale. This fact has been attributed to the fact that the PIB used in the solvent binds to nearest neighbor fumed silica particles, sometime permanently creating on average larger aggregates, and corroborated by Dullaert and Mewis [26] [30] [31] [32]. A cartoon of this phenomena is shown in Figure 2. The cartoon shows that the smaller sized constitutive raw particles bind together, permanently to form larger particles, which then form larger structures by interacting, and bonding with each other [30]. SANS probes length scales of $\sim 100-1000 \AA ̊$ over which the microstructure does not change with flow [1].

Figure 1(a) shows the quiescent scattering pattern, then scattering patterns at four different steady state shear rates in the top row of images. The different shear rates are clearly depicted in Figure 1(b) with numbers inserted that correlate to each of the images of the scattering at different shear rates in Figure 1(a). While the bottom row shows nearly identical difference patterns calculated by subtracting the quiescent patter (first image in first row of Figure 1(a)). This shows that the length scales of the agglomerates in the $2.9 \mathrm{vol} \%$ fumed silica in paraffin oil and PIB are too large to be detected by neutron scattering, therefore suggesting a transition to light scattering for better elucidation of the microstructure. Figure 2 below shows a cartoon (a) of the microstructure along with a TEM image (b) of the aggregates [1].

As neutron scattering cannot detect changes in our fumed silica microstructure 


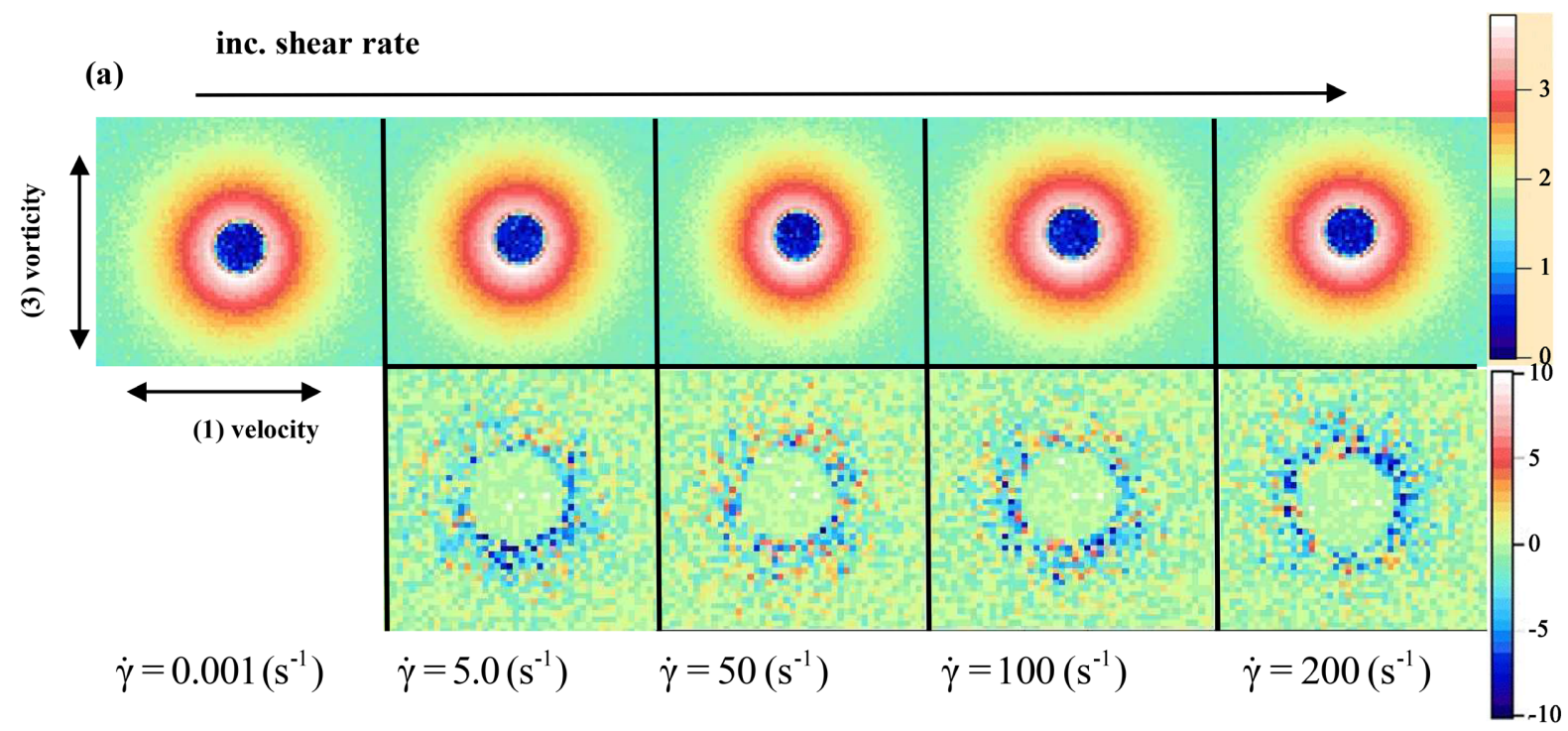

(1)

(2)

(3)

(4)

(5)

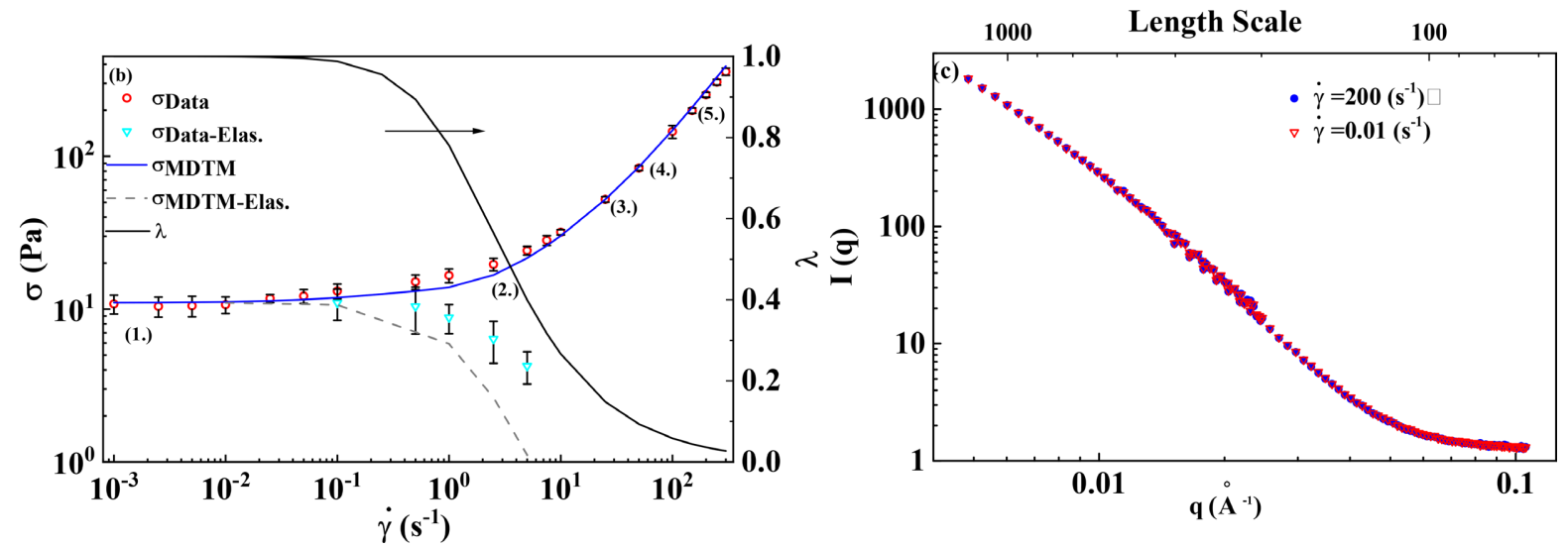

Figure 1. (a) 2-dimensional small angle neutron scattering (SANS) patterns (upper row) in the (1,3) plane and difference patterns bottom row (by subtracting the quiescent scattering pattering shown in (1), $\quad \dot{\gamma}=0.001 \mathrm{~s}^{-1}$ ); (2) $\dot{\gamma}=5.0 \mathrm{~s}^{-1} ;(3) \quad \dot{\gamma}=50.0 \mathrm{~s}^{-1} ;(4)$ $\dot{\gamma}=100.0 \mathrm{~s}^{-1}$; (5) $\dot{\gamma}=200.0 \mathrm{~s}^{-1}$; (b) Steady state flow curve depicting shear rates and locations of SANS difference patterns calculated; (c) $I(q)$ vs. $q\left(\AA^{-1}\right)$ calculated at two different shear rates listed in Figure 1.

(a)

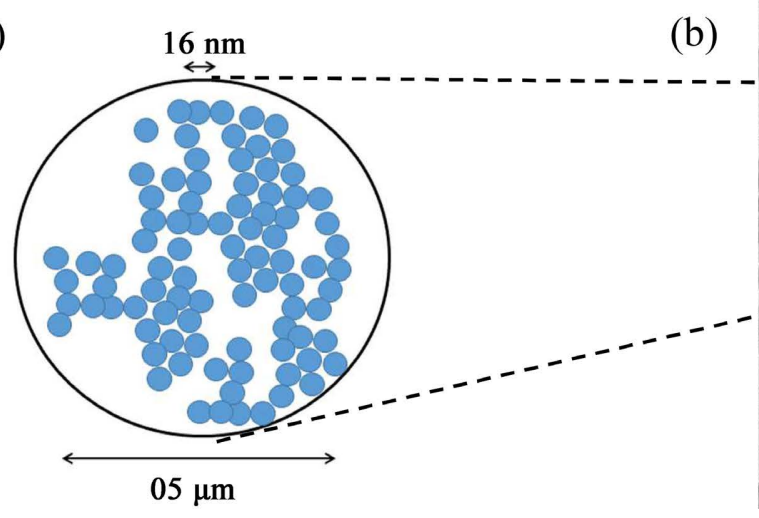

(b)

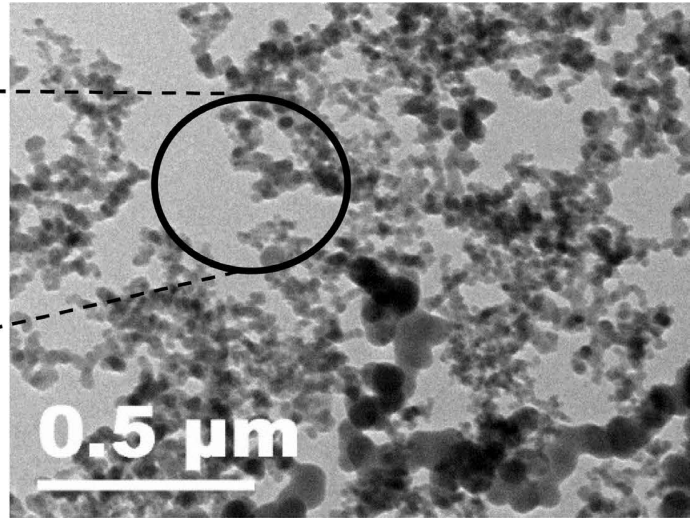

Figure 2. (a) Schematic of fumed silica aggregates; (b) TEM image of aggregates of 2.9 vol\% fumed silica in paraffin oil and PIB [1] [2]. 
under flow, larger length scales are probed by using small angle light scattering with the Rheo-SALS attachment to the DHR3 rheometer.

\subsection{Small Angle Light Scattering}

In this section the details of the Small Angle Light Scattering (SALS) data, and corresponding structural analysis are discussed. Starting with the calibration procedure for the SALS instrument using $3 \mu \mathrm{m}$ spheres in water [1].

First, the SALS calibration procedure shown pictorially in Figure 3 is described with the final correlated equations shown below the figure. The procedure involves using the $3 \mu \mathrm{m}$ calibration spheres provided in a dilute solution with water as the solvent. Then the images are taken with the Lumenera software [33] for comparison. As shown in the TA Instrument SALS literature the calibration sphere scattering pattern and the pure water scattering pattern are loaded into the ImageJ software package. Using ImageJ an image subtraction is performed to get the final image shown in Figure 3(c) [20] [21] [22] [23] [24]. Next the actual MiePlot [34] scattering pattern of the spheres is obtained from MiePlot, Figure 3(d) or the TA Instrument SALS literature [27] [35] [36]. With the final subtracted image, a radial profile calculation is performed with ImageJ, and the resulting output is shown in Figure 3(e). Finally, the two images must be parametrically matched. This is accomplished by marrying five key points in both images: the zero point; the known location of the beamstop, the first valley; the first peak; and the approximate location of second valley; all labeled below with dashed lines, this will correlate the number of pixels to our $q$ range, and, using MATLAB, can generate the conversions between pixels and $q$. A second order polynomial is used showing good agreement in Figure 3(g) and shown here in Equation (13),

$$
q(\text { pix })=-5.20 \mathrm{e}^{-6}(\text { pix })^{2}+1.25 \mathrm{e}^{-2}(\text { pix })+2.36 \mathrm{e}^{-2} .
$$

Additionally, using the known refractive index of water, 1.33 pixels can be correlated to scattering angle $\theta$ Equation (14),

$$
\theta(p i x)=2.57 \mathrm{e}^{-5}(\text { pix })^{2}+5.04 \mathrm{e}^{-2}(\text { pix })+1.35 \mathrm{e}^{-1} .
$$

However, when transitioned to the analysis of the fumed silica particles, the shift to the average refractive index of our pure solvent 1.48 must be accounted for via the following relationship between the scattering vector $\boldsymbol{q}$, with scattering angle, $\theta$, Equation (15),

$$
\boldsymbol{q}=\frac{4 \pi n}{\lambda_{0}} \sin \left(\frac{\theta}{2}\right),
$$

where $n$ is the refractive index of the pure solvent ( 1.33 for water, and 1.48 for paraffin oil/PIB) and $\lambda_{0}$ is the wavelength of the laser $(0.635 \mu \mathrm{m})$ [20] [21] [22] [23] [24]. The pixel to $\boldsymbol{q}$ is correlated with Equation (13), and the intensity must also be correlated first with a correction shown in Equation (16) below,

$$
I(\boldsymbol{q})_{\operatorname{corr}}=\frac{I(\boldsymbol{q})}{\cos (\theta)^{3}},
$$




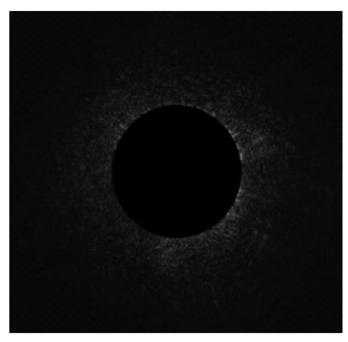

(a)

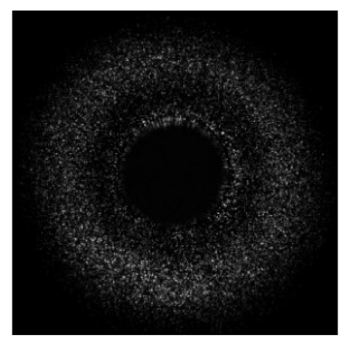

(b)

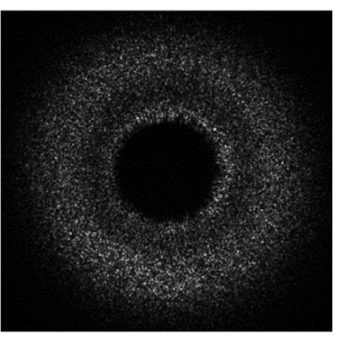

(c)
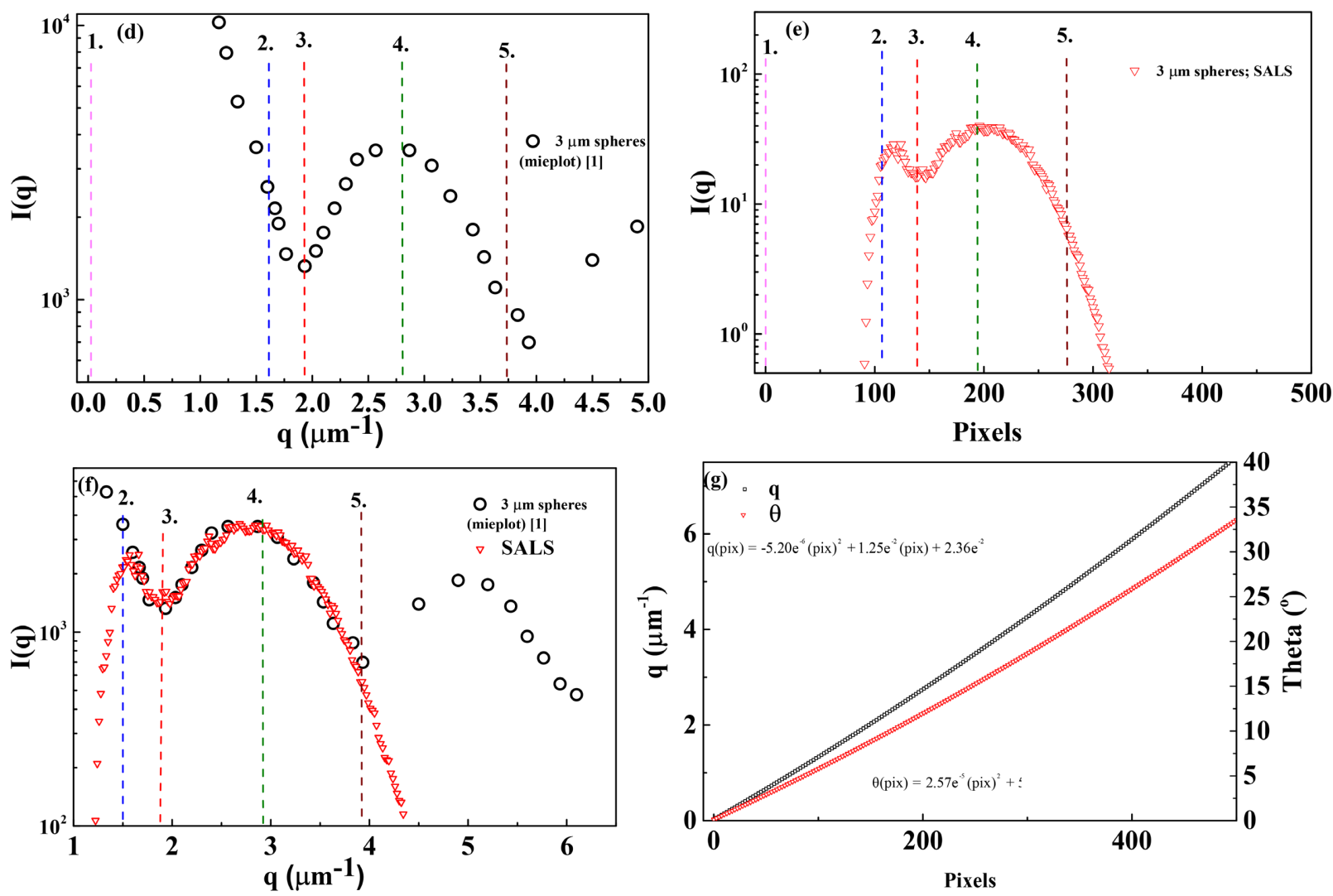

Figure 3. (a) Small angle light scattering (SALS) pattern of $3 \mu \mathrm{m}$ spheres in water; (b) SALS of pure water; (c) SALS pattern of pure water subtracted from $3 \mu \mathrm{m}$ spheres in water with ImageJ software; (d) MIE scattering of $3 \mu \mathrm{m}$ spheres (units of Intensity vs. q); (e) SALS scattering pattern of $3 \mu \mathrm{m}$ spheres from (c) in ImageJ (units of Intensity vs. Pixels), where line 1. is the beamstop, line 2. is the first trough and line 3. is the first peak; (f) Calibrated results showing overlapping maximum intensity, and placement of first peak; $(\mathrm{g})$ results showing parametric relationship between number of pixels and scattering vector, $\boldsymbol{q}$, and number of pixels and scattering angle [1] [20] [32].

and then using a multiplier, which for this data is 85 , in such a way as to show overlap with the MiePlot scattering pattern [20] [21] [22] [23] [24]. Lastly, the conversion between the $\boldsymbol{q}$ vector and the size scale of the aggregates is shown in Equation (17),

$$
l=\frac{2 \pi}{\boldsymbol{q}} .
$$

Using the calibration of pixels to $q$ one can prosecute the analysis of the SALS images taken under steady state and LAOS conditions [1]. A few more points 
first: 1) To ensure consistency in analysis the size of the images used for analysis must conform to the size of the calibration images $(1280 \times 1024$ pixels $)$; 2$)$ The pure solvent scattering must also be subtracted from all images to be analyzed shown in Figure 5. The Snag-It screen capture software can facilitate and required resizing of images [23]. We close by reiterating that the correlations between pixels and $q$, Equation (13), as well as the conversion to $\theta$, Equation (15), and the intensity correction Equation (16), and multiplier must be used consistently to quantitatively analyze the light scattering data [1].

\section{Materials and Methods}

In this section the fitting procedure is described and shown via the steady state SALS results. The best model as demonstrated convincingly by Armstrong and co-workers (2016) is used to simultaneously fit the steady state flow curve and two sets of LAOS data at $\omega=1(\mathrm{rad} / \mathrm{s}), \quad \gamma_{0}=1 \& 10$ with the best fit parameter results over 15 trials shown in Table 1 , and the correlation matrix in Table 2. Here, it is best to use the best fit parameter results from [2] as the initial guess.

\subsection{Experimental Protocol}

The steady state and LAOS data was collected on a Discovery Hybrid Rheometer - 3 (DHR-3), stress-controlled rheometer by TA Instruments at a temperature of $25^{\circ} \mathrm{C}$. Due to the thixotropic nature of the material a pre-shear was conducted prior to each new shear rate tested for $300 \mathrm{~s}$ at $300 \mathrm{~s}^{-1}$. The DHR-3 with SALS attachment is shown below in Figure 6. The scattering pattern at each of the shear rates and during the LAOS experiments were recorded with the software MiePlot and ImageJ [33] [34] [35] [36]. In the LAOS experiments the strain is given as $\gamma(t)=\gamma_{0} \sin (\omega t)$, and the shear rate, the first time derivative of strain as $\dot{\gamma}(t)=\gamma_{0} \omega \cos (\omega t)$, where $\gamma_{0}$ is the strain amplitude and $\omega$ is the frequency of oscillation.

Since the SALS can only be used with the DHR-3 stress-controlled instrument, it was necessary to perform the rheological tests in pseudo-strain-controlled mode, with a quartz, parallel plate geometry causing the data signal to pick up a large noise signal. Two problems arise because the DHR-3 is a stress-controlled instrument with the ability to operate in strain-controlled mode: This instrument makes use of an additional control loop around the torque, which is adjusted accordingly to maintain the programed strain. This additional feedback loop, in conjunction with an oscillating LAOS flow induces a very noisy signal. To demonstrate that the signals are approximately equal, both sets of data were plotted simultaneously in dimensional form in Figure 4.

Due to the SALS instrument the laser aperture is set approximately $75 \%$ from the center of the quartz, plate geometry. Additionally, because the geometry used does not have a homogeneous shear field the shear rate at the rim of the geometry is different than that within the rheological experiment between the flat plates. To replicate the rheology experiments as produced on the ARESG2 


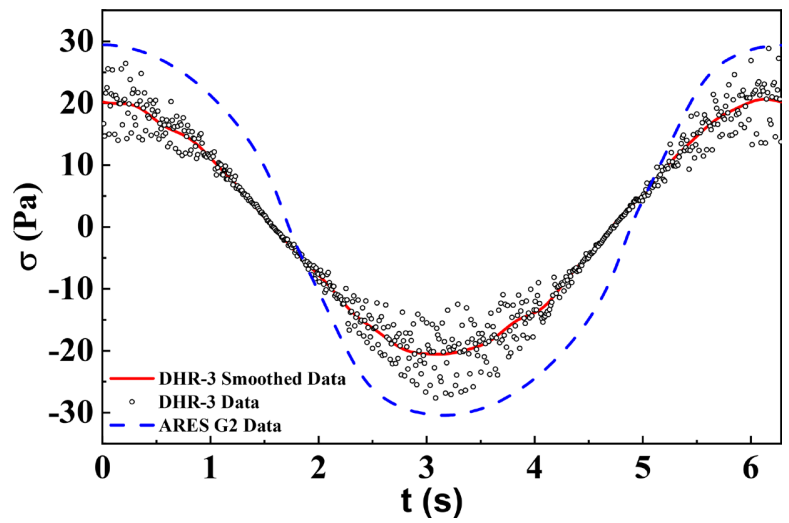

Figure 4. DHR3 and ARESG2 LAOS data comparison at $\omega=1(\mathrm{rad} / \mathrm{s}) ; \gamma_{0}=10$ [1].

instrument the shear rate must be identical at the aperture. Therefore, the shear rate at the rim is set to be $133 \%$ that which is required at the laser aperture.

\subsection{Model Fitting Procedure}

The model fitting procedure incorporated to fit the steady state data and two sets of large amplitude oscillatory shear data to the MDTM parameters was the parallel tempering algorithm here [4]. An identical procedure is outlined here [4], whereby the steady state parameters of the model are fitted to the steady state data, then holding those parameters constant, fitting the transient parameters [4]. Since the steady state data was spread over three orders of magnitude of stress a normalized cost function was implemented, while fitting the transient parameters implemented a dimensional formulation of the cost function [1] [2] [4] [36] [37] [38].

\section{Results and Discussion}

\subsection{MDTM Fitting to Data}

From Table 1, the parameters of the MDTM are correlated in some instances just as in the fitting of the steady state and transient data shown here [1] [2]. Table 1 shows the parameters, gives the parameter definition, provides the average and standard deviation of each parameter over the 15 iterations of fitting, and the best parameter values. Here the second thixotropic time constant $t_{r 2}$ is correlated negatively with $a, n_{2}$, and $\eta_{S T}$. Table 2 shows the model parametric correlations. The parameter $k_{G}$, and $t_{r}$, are positively correlated and $a$ is also positively correlated with $n_{2}$. The correlations between parameters indicate that there is not a single unique solution, and that the various parameters shift their values to best fit the data, and although in this case there appears to be a single "basin" for the best parameters. With the set of parameters fit only to two sets of LAOS and the steady state flow curve, the same parameters values can now be compared as determined in [2] where 17 sets of transient step-ups and step-down and the steady state flow curve are fit. In the case of the LAOS data fitting there are some interesting changes to the fit parameters. The clearest is the striking decrease in 
$t_{r 2}$, which is the shear aggregation time constant, and the decrease of $k_{B r o w n}$. The other parameters of the model were of the same order of magnitude and had similar values $( \pm 10 \%)$. This implies that to fit the LAOS data to the MDTM the structure evolution parameters had to be modified. As has been seen in literature with other systems [14] and the light scattering, the structure and structure dynamics are different between steady state flow, and LAOS flow [7].

The correlation matrix shown in Table 2, below highlights green all parametric correlations between the MDTM parameters.

\subsection{Steady State Analysis}

Below we show the schematic of the Azimuthal Distribution Function (ADF)

Table 1. MDTM Parameters fit to steady state and two sets of LAOS [1].

\begin{tabular}{|c|c|c|c|c|c|c|c|}
\hline Par. & Units & Meaning & Range Initial Guess & Limiting Vals. & Optimal & $\mu:$ & $\sigma:( \pm)$ \\
\hline$\sigma_{y 0}$ & $\mathrm{~Pa}$ & Yield stress & $(-)$ & $(-)$ & 11 & $(-)$ & $(-)$ \\
\hline$\eta_{\infty}$ & $\mathrm{Pa} \mathrm{s}^{\mathrm{n} 1}$ & Infinite shear viscosity & $(-)$ & $(-)$ & 1.17 & $(-)$ & $(-)$ \\
\hline$n_{1}$ & $(-)$ & Power law $\eta_{\infty}$ & $(-)$ & $(-)$ & 1 & $(-)$ & $(-)$ \\
\hline$G_{0}$ & $\mathrm{~Pa}$ & Elastic Modulus & $(-)$ & $(-)$ & 450 & $(-)$ & $(-)$ \\
\hline$m$ & $(-)$ & Power law par. of $\gamma_{e}$ evol. & $(-)$ & $(-)$ & -1.5 & $(-)$ & $(-)$ \\
\hline$K_{S T}$ & $\mathrm{~Pa} \mathrm{~s} \mathrm{~s}^{\mathrm{n} 2}$ & Consistency par. & {$[9-11]$} & $>0$ & 10.75 & 9.23 & 1.890 \\
\hline$n_{2}$ & $(-)$ & Power law par. $K_{S T}$ & {$[0.5-1]$} & {$[0.5-1]$} & 0.89 & 0.71 & 0.159 \\
\hline$a$ & $(-)$ & Power law par. shear break. & {$[1-2]$} & {$[1-2]$} & 1.29 & 1.18 & 0.254 \\
\hline$d$ & $(-)$ & Power law par. shear aggr. & {$[0.25-0.75]$} & {$[0.25-0.75]$} & 0.43 & 0.60 & 0.211 \\
\hline$k_{\text {Brown }}$ & $\mathrm{s}^{-1}$ & Char. time of Brownian mot. & {$\left[1 \mathrm{e}^{-3}-1\right]$} & $>0$ & 0.10 & 0.23 & 0.111 \\
\hline$t_{r 1}\left(k_{\text {break }} / k_{\text {Brown }}\right)$ & $\mathrm{s}^{\mathrm{a}}$ & Char. time of $\lambda$ breakdown & {$\left[1 \mathrm{e}^{-3}-1\right]$} & $>0$ & 0.42 & 0.23 & 0.234 \\
\hline$t_{r 2}\left(k_{\text {aggr }} / k_{\text {Brown }}\right)$ & $s^{\mathrm{d}}$ & Char. time of $\lambda$ buildup & {$\left[1 \mathrm{e}^{-3}-1\right]$} & $>0$ & 0.59 & 1.31 & 0.617 \\
\hline$k_{G}$ & $\mathrm{~s}^{-1}$ & Char. time of G evol. & {$\left[1 \mathrm{e}^{-3}-1\right]$} & $>0$ & 0.04 & 0.05 & 0.021 \\
\hline
\end{tabular}

Table 2. Correlation matrix of parameters fit to steady state flow curve and two sets of LAOS (p-value in parenthesis) [1].

\begin{tabular}{|c|c|c|c|c|c|c|c|c|}
\hline & $t_{r 1}$ & $t_{r 2}$ & $k_{\text {Brown }}$ & $a$ & $d$ & $n_{2}$ & $\eta_{S T}$ & $k_{G}$ \\
\hline$t_{r 1}$ & 1.00 & & & & & & & \\
\hline$t_{r 2}$ & 0.27 & 1.00 & & & & & & \\
\hline$k_{\text {Brown }}$ & -0.40 & 0.46 & 1.00 & & & & & \\
\hline$a$ & -0.21 & $-0.53(0.042)$ & -0.20 & 1.00 & & & & \\
\hline$d$ & 0.41 & -0.19 & -0.44 & 0.06 & 1.00 & & & \\
\hline$n_{2}$ & -0.17 & $-0.62(0.0127)$ & -0.10 & $0.79(0.0005)$ & -0.21 & 1.00 & & \\
\hline$K_{S T}$ & 0.32 & $-0.562(0.029)$ & $-0.75(0.0011)$ & 0.41 & 0.22 & 0.39 & 1.00 & \\
\hline$k_{G}$ & $0.695(0.0039)$ & 0.11 & -0.30 & -0.49 & 0.46 & -0.39 & -0.03 & 1.00 \\
\hline
\end{tabular}


calculation that we use to calculate the alignment factor over a specific $q$ range. This is depicted in Figure 5(a), Figure 5(b), and shows how the intensity is calculated vs. the angle $\theta$ from -180 to 180 . For our calculations Equation (11) uses $\theta_{0}=0$. The intensity is averaged at each increment of the entire $\theta$ range, within the narrow $q$ region outlined below. Our $q$ region is $q=1.9 \mu \mathrm{m}^{-1}-2.3 \mu \mathrm{m}^{-1}$, and using Equation (15) the length scale within the region is $2.7 \mu \mathrm{m}-3.3 \mu \mathrm{m}$. The $I(\boldsymbol{q})$ vs. $\theta$ data is then used to calculate the alignment factor using Equation (16). Numerical experiments were conducted to find the best numerical integration technique, to include fitting a trigonometric function of the form shown here to fit the $I(\boldsymbol{q})$ data,

$$
f(\theta)=A \cos (2 \theta)+B
$$

Figure 5(a), Figure 5(b) show in schematic for how the ADF is calculated, using the average intensity of a $\Delta q$ range from $-180^{\circ}$ to $180^{\circ}$. For our ADF calculations the $q$ range is $1.9 \mu \mathrm{m}^{-1}$ to $2.3 \mu \mathrm{m}^{-1}$, and the background of each set of steady state scattering data is subtracted out. The background of each steady state scattering pattern is estimated from the raw scattering data with the background $(\mathrm{bkg}) \sim \min (I(q))$. Figure 5(c), Figure 5(d) show the results of our first simple ADF fitting procedure using Equation (18). A quick analysis of the fits shows that the fits are not accurately capturing the scattering pattern. This approach was not as accurate as a higher order numerical integration scheme like

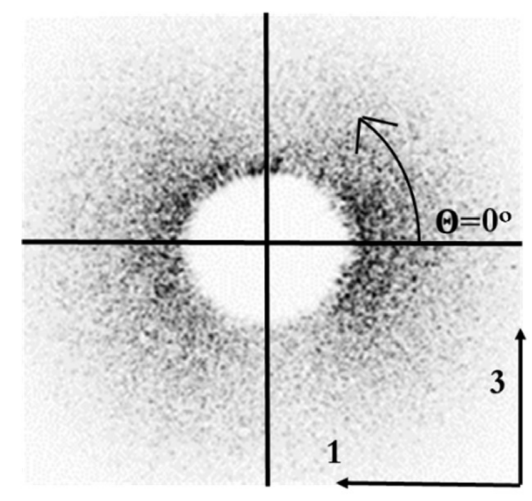

(a)

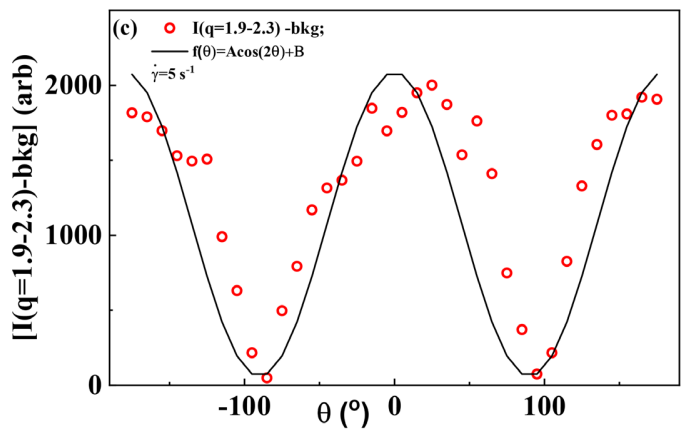

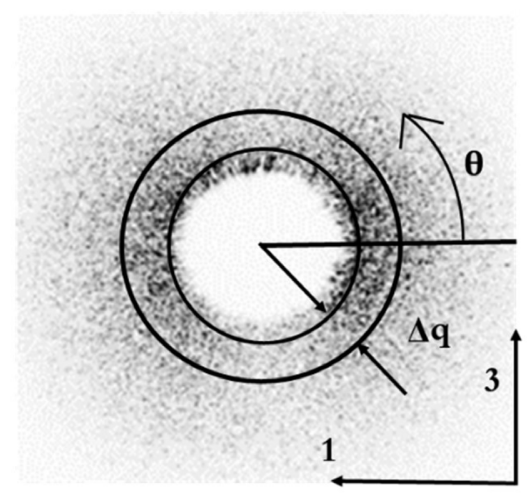

(b)

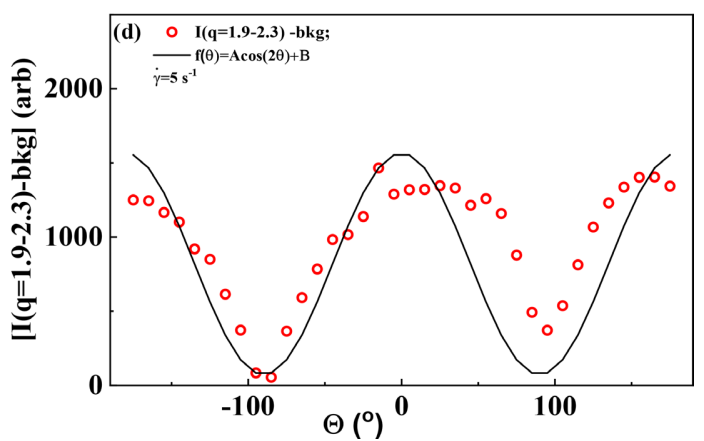

Figure 5. (a) Azimuthal Distribution Function (ADF) Schematic; (b) ADF Schematic depicting $\Delta q$; (c) Simple fitting to Equation (18); $A=746$ (arb), $B=818(\mathrm{arb})$ at $\dot{\gamma}=200 \mathrm{~s}^{-1}$; (d) Simple fitting to Equation (18); $A=1590$ (arb), $B=1423$ (arb) at $\dot{\gamma}=5 \mathrm{~s}^{-1} \quad[1]$. 
Simpson's composite integration formula. It is noted here that for the alignment factor calculations the background light scattering was subtracted out a priori. With the $A$, and $B$ parameters estimated it is now possible to calculate the alignment factor, $A_{f}$ shown in Equation (11). Using this technique, the calculated value of the alignment factor for the two shear rates shown in Figure 5(c), Figure $5(\mathrm{~d})$ is 0.49 and 0.45 , respectively. It is noted that there is not much change in the alignment factor at two shear rates at different orders of magnitude. Although the calculation shows that there is more anisotropy in the $\dot{\gamma}=5 \mathrm{~s}^{-1}$ system, as is expected because at the higher shear rate the structure has been destroyed. Because the values are so close, and there is obvious error introduced in our simple fitting of Equation (18), we are transitioning to a more robust, numerical integration procedure of the data directly using the Composite Trapezoid, and Composite Simpson's rule.

The mapping of planes is shown in Figure 6 and annotated in Figure 7(a). Using this graphic representation of the mapping one can see how the $(1,3)$ plane relates to the final scattering images from ImageJ. The $(1,3)$ plane is the default, and only setting for use with the SALS instrument [20] [21].

We show in Figure 7 (a) a series of raw 2-dimensional scattering images in order of increasing shear rate at steady state. Below each image is the same image but after subtracting the quiescent image at zero shear rate, and adding a color scheme, to demonstrate that there is a change in scattering, implying some correlated change in microstructure. Above each raw image is the inverse image, shown to assist in discerning scattering differences. By following from low to high shear rate, one can discern a developing, then decreasing butterfly pattern, characteristic of non-isotropic aggregates or particles, and showing some evolution of microstructure [19]. A similar result here has been previously reported by [14] [15], using SANS on a colloidal gel [7] [35]. In Figure 7(c), Figure 7(d) we show the azimuthal distribution function over our $q$ range, and the $I(q)$ vs. $q$ at $\Theta=0$ (in the flow direction) respectively. Figure $7(\mathrm{e})$ is the structure parameter predictions using the MDTM, with the $A_{f}$ calculation. According to our

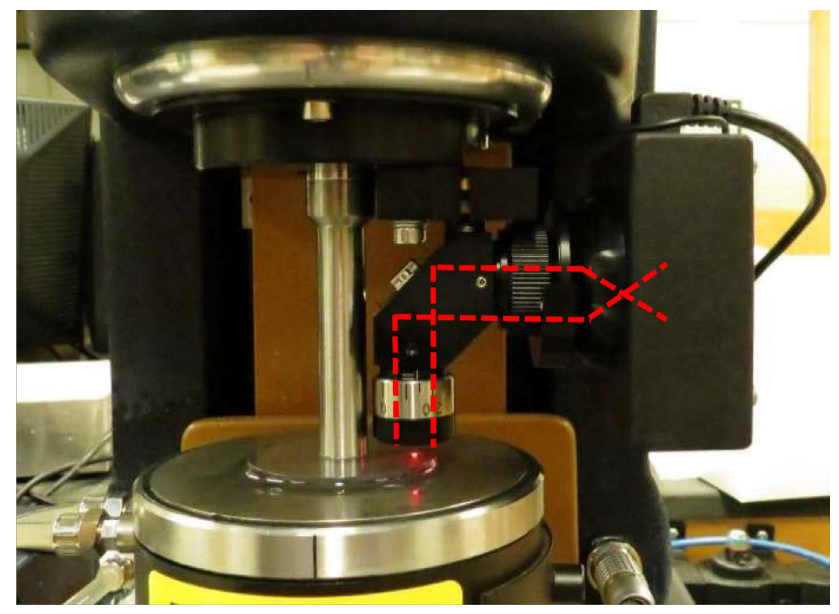

Figure 6. Image of the SALS Setup with Mapping to Show (1,3) plane [1]. 
inc. shear rate

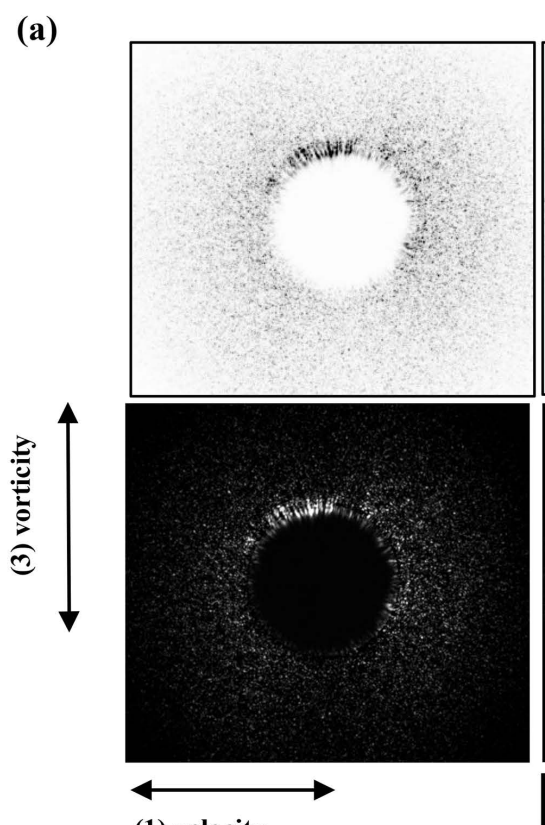

(1) velocity

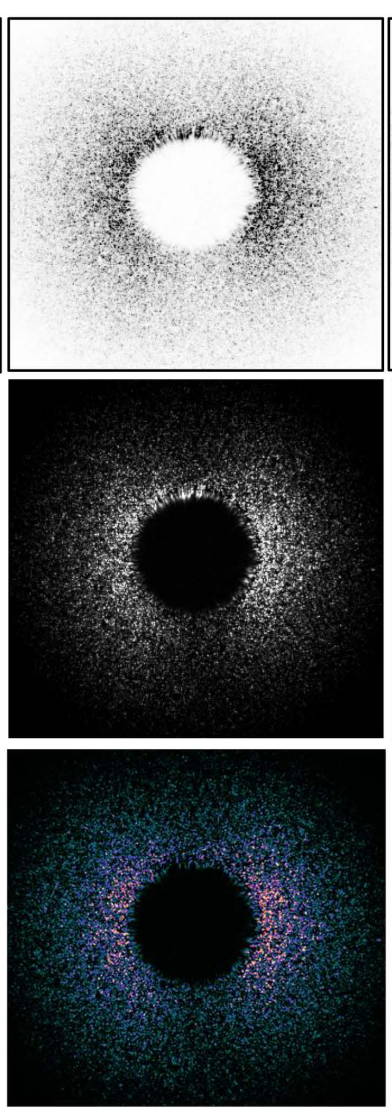

(2)

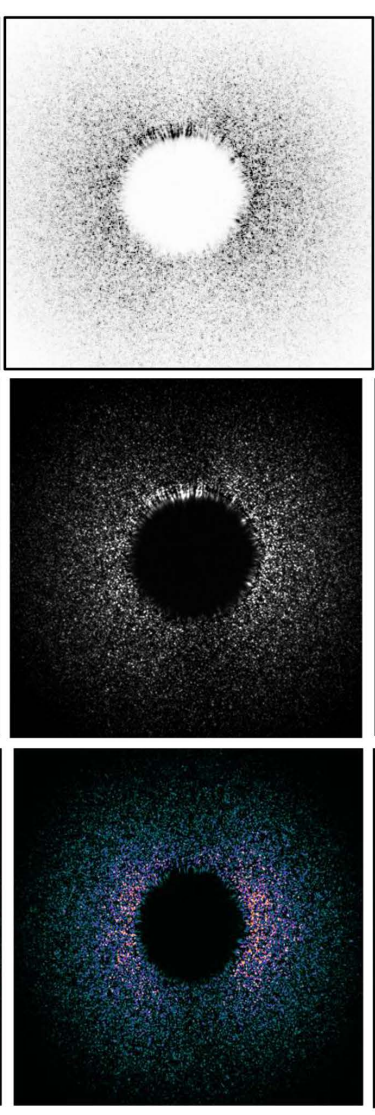

(3)

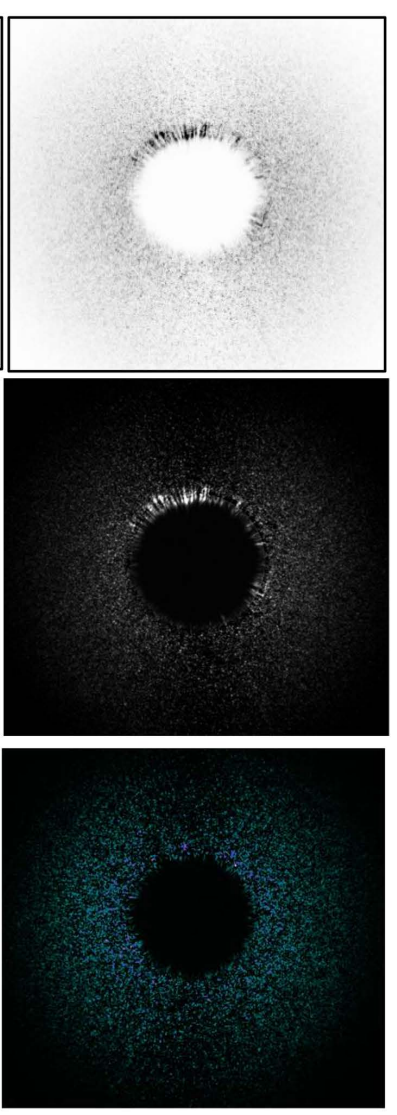

(4)

inc. shear rate
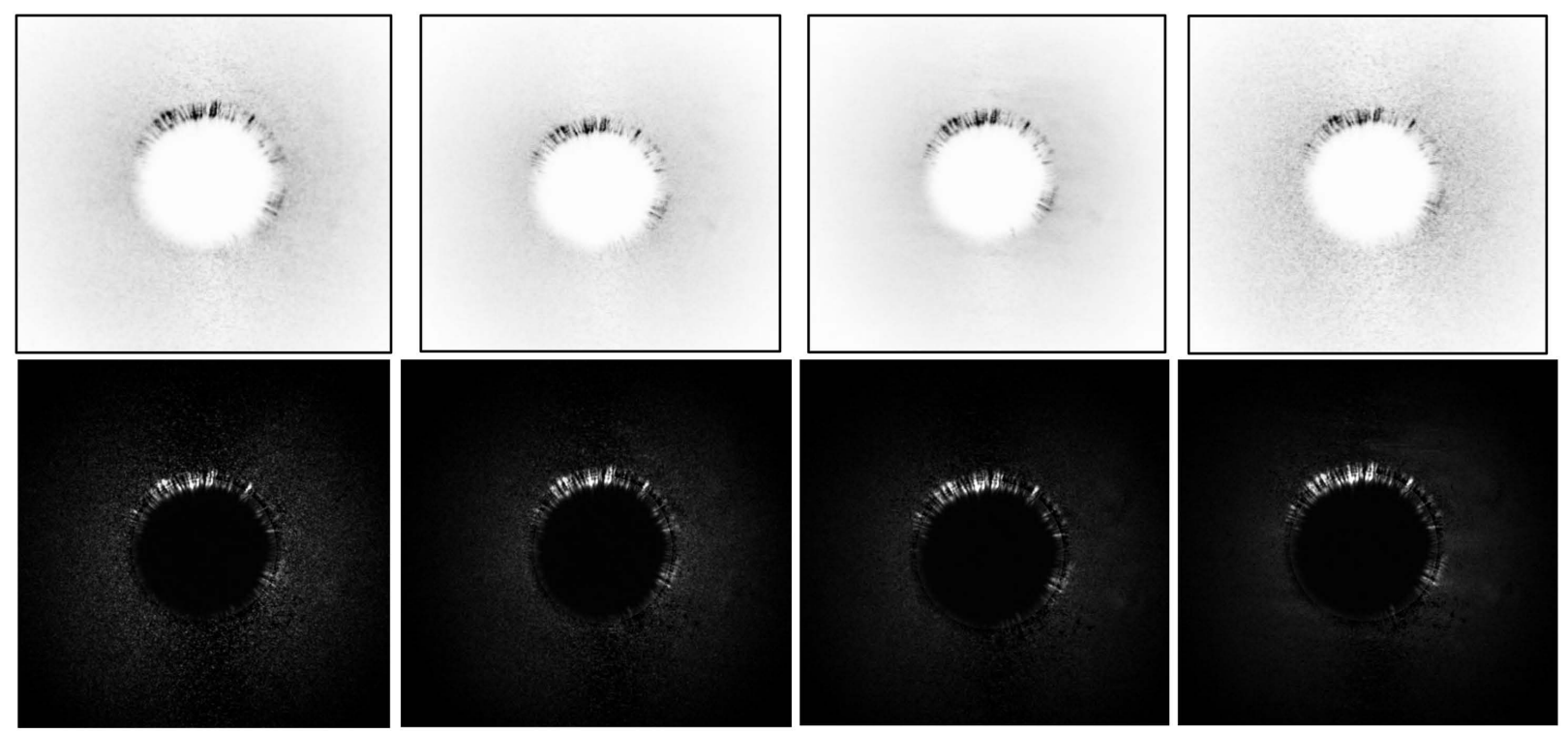


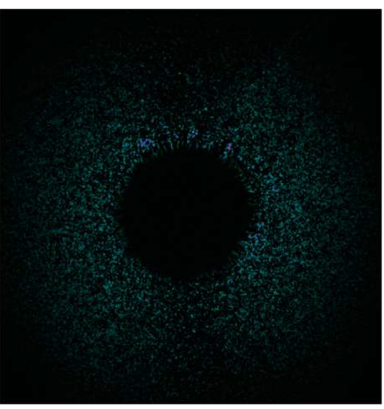

(5)

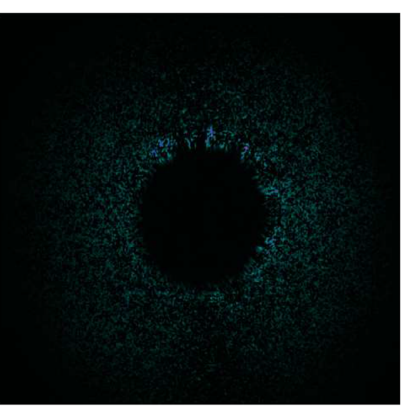

(6)

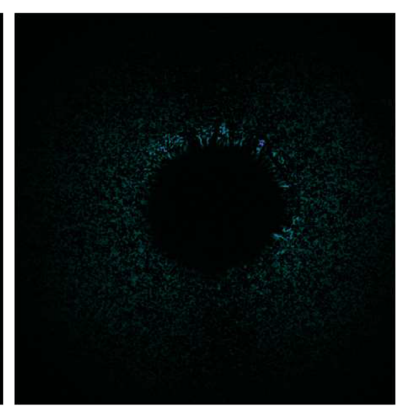

(7)

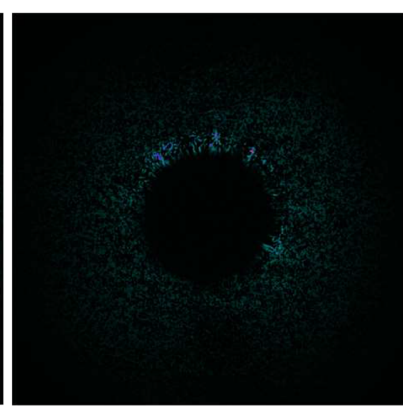

(8)
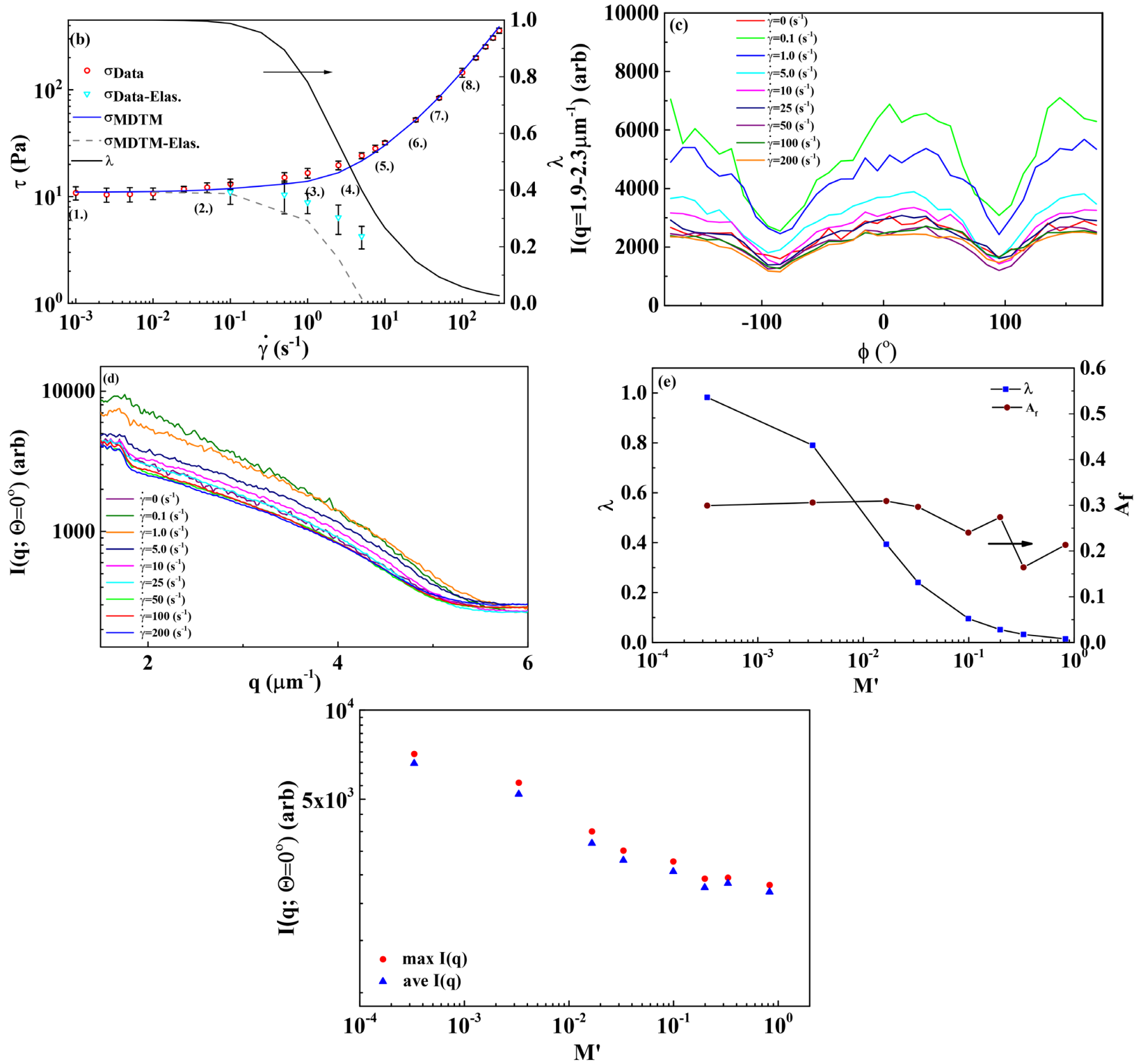

Figure 7. (a) No. 1-9 top row: small angle light scattering in the $(1,3)$ plane inverse images; middle row: small angle scattering in $(1,3)$ plane patterns at steady shear rates shown in (a); Bottom row: small angle difference scattering patterns (by subtracting image 0) ; (b) Steady state flow curve with shear rates of SALS scattering images depicted; (c) Azimuthal distribution functions (ADF) taken at $q$ range $1.9-2.3 \mu \mathrm{m}^{-1}$ (d) $I\left(q ; \theta=0^{\circ}\right)$ vs. $q\left(\mu \mathrm{m}^{-1}\right)$ for 8 shear rates; (e) plot of $\lambda$ and $A_{f}$ vs. $M^{\prime}$ together; (f) Maximum and average intensity vs. $M^{\prime}$. (Legend shown in Table 3 ) [1]. 
Table 3. Legend to interpret Figure 7 [1].

\begin{tabular}{ccccc}
\hline No. & Shear Rate $\left(\mathrm{s}^{-1}\right)$ & $A_{f}$ & $\lambda$ & $M^{\prime}$ \\
\hline 1 & 0 & 0.25 & 1.00 & 0.00000 \\
2 & 0.1 & 0.30 & 0.98 & 0.00033 \\
3 & 1 & 0.31 & 0.79 & 0.00331 \\
4 & 5 & 0.31 & 0.39 & 0.01655 \\
5 & 10 & 0.30 & 0.24 & 0.03310 \\
6 & 25 & 0.24 & 0.10 & 0.09929 \\
7 & 50 & 0.27 & 0.05 & 0.19858 \\
8 & 100 & 0.16 & 0.03 & 0.33097 \\
9 & 200 & 0.21 & 0.01 & 0.82743 \\
\hline
\end{tabular}

prediction of $\lambda$ and $A_{f}$ calculation the two are correlated, in that the trend for both is a decrease with respect to shear rate, and $M$ ' Figure 7 (f) shows the average and maximum intensity for each of our steady shear rates. The trend is clear, increasing shear rate, after the initial rise after $\dot{\gamma}=0$, causes less scattering, due primarily to less structure, due to the structure breakage caused by the increase in shear rate.

Figures $7(\mathrm{a})-(\mathrm{e})$ show interesting features. Here we have plotted the new fit for the steady state flow curve with the parameters from Table 3 , and the $\lambda$ predictions. We are also showing the azimuthal distribution functions at several the steady state shear rates using Equation (12). Here we see that there is an initial increase in the intensity at low shear rates but that this effect dies down as the shear rate increases above further from $1 \mathrm{~s}^{-1}$. The $q$ range for the azimuthal distribution function is $1.9-2.7 \mu \mathrm{m}^{-1}$ which corresponds to a length scale of 2.7 $3.3 \mu \mathrm{m}$ which was calculated using Equation (17) [1]. The $I(q)$ vs. $q$ curves also show the same trend exhibited in the azimuthal distribution function. But the most telling and informative observation from the steady state rheology and scattering is the $\lambda$ and $A_{f}$ vs. $M^{\prime}$ curve which indicates that there does not seem to be any correlation between our steady state flow condition effect on $\lambda$ and $A_{f}$. This could simply mean that to induce a change in $A_{f}$ with this model thixotropic system one must first have oscillating flow, whereby the lambda value is greatly affected by the magnitude of the shear rate [1].

\section{Conclusions}

Here we have shown a unique ability to simultaneously probe the microstructure of the model thixotropic system while performing rheological tests, thusly incorporating all four corners of the soft matter interrogation tetrahedron. Additionally, we started by demonstrating that comparing $\mathrm{A}_{\mathrm{f}}$ calculated using neutron scattering data and simultaneously using the MDTM to predict $\lambda$ was possible, and useful to make relevant correlations of how the flow field affects both 
measures of a material's microstructure [1] [2] [11]-[17] [37] [38]. This method was subsequently applied to 2.9 vol\% fumed silica in paraffin oil and PIB. While the MDTM model proved suitable to the experimental purpose, it necessitated a transition of the utilized scattering source to small angle light scattering because of the larger length scale of these aggregates [1].

Upon transitioning to the small angle light scattering experiments, we were able to see and discern key changes in the microstructure for steady state and LAOS flow at varying shear rates. With our scattering images and Image J were able to use the azimuthal averaging, and radial profile options to calculate alignment factor. We also used our best scalar thixotropic model, the MDTM to fit the model directly to the steady state and LAOS, thusly predicting the structure $\lambda$. This now offered a vehicle to make correlations between the alignment factor and structure to look for patterns. We discovered that indeed there were correlations, although barely discernable, but only at strain amplitude 1 and 10, while at strain amplitude 100 it appeared that the structure was too broken down to be able to use the alignment factor, as its magnitude was greatly diminished. It also noteworthy that the structure as predicted by the MDTM does seem to correlate more with the maximum scattering intensity, which is another metric of a structured material state. We have successfully shown a correlation between our scalar structure parameter as predicted by the MDTM and the alignment factor of the microstructure in a 2.9 vol\% fumed silica in paraffin and PIB.

\section{Acknowledgements}

The authors acknowledge the support and funding assistance from the U.S. Army, and the Department of Chemistry and Life Science, United States Military Academy. The authors also acknowledge support in the form of helpful and insightful discussions with Dr. Norman Wagner, Dr. Antony Beris from University of Delaware. The authors acknowledge insightful discussions with Dr. Simon Rogers from the University of Illinois Urbana-Champagne. The views expressed herein are those of the authors and do not reflect the position of the United States Military Academy, the Department of the Army, or the Department of Defense.

\section{Conflicts of Interest}

The authors declare no conflicts of interest regarding the publication of this paper.

\section{References}

[1] Armstrong, M.J. (2015) Investigating and Modeling the Thixotropic Behavior, Rheology of Complex Material. Ph.D. Thesis, University of Delaware, Newark.

[2] Armstrong, M.J., Beris, A.N., Rogers, S.A. and Wagner, N.J. (2016) Dynamic Shear Rheology of a Thixotropic Suspension: Comparison of an Improved Structure-Based Model with Large Amplitude Oscillatory Shear Experiments. Journal of Rheology, 60, 433-450. https://doi.org/10.1122/1.4943986 
[3] Armstrong, M.J., Beris, A.N., Rogers, S.A. and Wagner, N.J. (2017) Dynamic Shear Rheology and Structure Kinetics Modeling of a Thixotropic Carbon Black Suspension. Rheologica Acta, 56, 811-824. https://doi.org/10.1007/s00397-017-1038-8

[4] Armstrong, M.J., Beris, A.N. and Wagner, N.J. (2016) An Adaptive Parallel Tempering Method for the Dynamic Data-Driven Parameter Estimation of Nonlinear Models. AIChE Journal, 63, 1937-1958. https://doi.org/10.1002/aic.15577

[5] Rogers, S.A. (2012) A Sequence of Physical Processes Determined and Quantified in LAOS: An Instantaneous Local 2D/3D Approach. Journal of Rheology, 56, 11291151. https://doi.org/10.1122/1.4726083

[6] Rogers, S.A. (2017) In Search of Physical Meaning: Defining Transient Parameters for Nonlinear Viscoelasticity. Rheologica Acta, 56, 501-525.

https://doi.org/10.1007/s00397-017-1008-1

[7] Lee, C.W. and Rogers, S.A. (2017) A Sequence of Physical Processes Quantified in LAOS by Continuous Local Measures. Korea-Australia Rheology Journal, 29, 269279. https://doi.org/10.1007/s13367-017-0027-x

[8] Gurnon, A.K. and Wagner, N.J. (2015) Microstructure and Rheology Relationships for Shear Thickening Colloidal Dispersions. Journal of Fluid Mechanics, 769, 242-276. https://doi.org/10.1017/jfm.2015.128

[9] Lopez-Barron, C.R., Wagner, N.J. and Porcar, L. (2015) Layering, Melting, and Recrystallization of a Close-packed Micellar Crystal under Steady and Large-amplitude Oscillatory Shear Flows. Journal of Rheology, 59, 793-820. https://doi.org/10.1122/1.4917542

[10] Helgeson, M.E., Vasquez, P.A., Kaler, E.W. and Wagner, N.J. (2009) Rheology and Spatially Resolved Structure of Cetyltrimethylammonium Bromide Wormlike Micelles through the Shear Banding Transition. Journal of Rheology, 53, 727-756. https://doi.org/10.1122/1.3089579

[11] Helgeson, M.E. (2009) Structure, Rheology, and Thermodynamics of Wormlike Micelle-Nanoparticle Mixtures. Ph.D. Thesis, University of Delaware, Newark.

[12] Lopez-Barron, C.R., Gurnon, A.K., Eberle, A.P.R., Porcar, L. and Wagner, N.J. (2014) Spatiotemporal Stress and Structure Evolution in Dynamically Sheared PolymerLike Micellar Solutions. Soft Matter, 10, 2889-2898. https://doi.org/10.1039/C3SM53113A

[13] Kim., J.M., Gurnon, K.A., Eberle, A.P., Porcar, L. and Wagner, N.J. (2014) The Microstructure and Rheology of a Model, Thixotropic Nanoparticle Gel Under Steady Shear and Large Amplitude Oscillatory Shear (LAOS). Journal of Rheology, 58, 13011328. https://doi.org/10.1122/1.4878378

[14] Eberle, A.P.R. and Porcar, L. (2012) Flow-SANS and Rheo-SANS Applied to Soft Matter. Current Opinion in Colloid and Interface Science, 17, 33-43.

https://doi.org/10.1016/j.cocis.2011.12.001

[15] Kim, J.M. (2013) Effect of the Range of Attraction on the Rheology, Microstructure, and Thermodynamics of Thermoreversible Gels with Adhesive Hard-Sphere Interactions. Ph.D. Thesis, University of Delaware, Newark.

[16] Gurnon, K.A. (2014) Nonlinear Oscillatory Rheology and Structure of Wormlike Micellar Solutions and Colloidal Suspensions. Ph.D. Thesis, University of Delaware, Newark.

[17] Walker, L.M. and Wagner, N.J. (1995) Structure of Isotropic Solutions of Rigid Macromolecules via Small-Angle Neutron Scattering: Poly (.gamma.-benzyl L-glutamate)/Deuterated Dimethylformamide. Macromolecules, 28, 5075-5081. https://doi.org/10.1021/ma00118a041 
[18] LaFollette, A.T. and Walker, L.M. (2011) Structural and Mechanical Hysteresis at the Order-Order Transition of Block Copolymer Micellar Crystals. Polymers, 3, 281-298. https://doi.org/10.3390/polym3010281

[19] Pignon, F., Magnin, A. and Piau, J.M. (1997) Butterfly Light Scattering Pattern Rheology of a Sheared Thixotropic Clay Gel. Physical Review Letters, 79, 4689-4692. https://doi.org/10.1103/PhysRevLett.79.4689

[20] Walker, L.M. (2009) Scattering from Polymer-Like Micelles. Current Opinion in Colloid and Interface Science, 14, 451-454. https://doi.org/10.1016/j.cocis.2009.08.001

[21] Behrens, M.A., Bergenholtz, J. and Pedersen, J.S. (2014) Temperature-Induced Attractive Interactions of PEO-Containing Block Copolymer Micelles. Langmuir, 30, 6021-6029. https://doi.org/10.1021/la500154s

[22] Agrawal, Y.C., Whitmire, A., Mikkelsen, O.A. and Pottsmith, H.C. (2008) Light Scattering by Random Shaped Particles and Consequences on Measuring Suspended Sediments by Laser Diffraction. Journal of Geophysical Research: Oceans, 113, C04023. https://doi.org/10.1029/2007JC004403.

[23] De Bruyn, J.R., Pignon, F., Tsabet, E. and Mignon, A. (2007) Micron-Scale Origin of the Shear Induced Structure in Laponite-Poly(Ethylene Oxide) Dispersions. Rheologica Acta, 47, 63-73. https://doi.org/10.1007/s00397-007-0211-x

[24] Gurnon, K. (2010-2012) LAOS of a 30nm Adhesive Hard Sphere System. GrenobleNIST.

[25] Pincot, A. and Armstrong, M. (2021) Novel Tensorial Thixo-Visco-Plastic Framework for Rheological Characterization of Human Blood. Scientific Reports, 11, Article No. 22004. https://doi.org/10.1038/s41598-021-01362-8.

[26] Dullaert, K. and Mewis, J (2006) A Structural Kinetics Model for Thixotropy. Journal of Non-Newtonian Fluid Mechanics, 139, 21-30.

https://doi.org/10.1016/j.jnnfm.2006.06.002

[27] Dimitriou, C.J., Ewoldt, R.H. and McKinley, G.H. (2013) Describing and Prescribing the Constitutive Response of Yield Stress Fluids Using Large Amplitude Oscillatory Shear Stress (LAOStress). Journal of Rheology, 57, 27-70. https://doi.org/10.1122/1.4754023

[28] Varchanis, S., Makrigiorgos, G., Moschopoulos, P., Dimakopoulos, Y. and Tsamopoulos, J. (2019) Modeling the Rheology of Thixotropic Elasto-Visco-Plastic Materials. Journal of Rheology, 63, 609-639. https://doi.org/10.1122/1.5049136

[29] Apostolidis, A., Armstrong, M.J. and Beris, A. (2015) Modeling of Human Blood Rheology in Transient Shear Flows. Journal of Rheology, 59, 275-298.

https://doi.org/10.1122/1.4904423

[30] Dullaert, K. (2005) Constitutive Equations for Thixotropic Dispersions. Ph.D. Thesis, Katholieke Universiteit Leuven, Leuven.

[31] Walker, L.M. and Wagner, N.J. (1996) SANS Analysis of the Molecular Order in Poly ( $\gamma$-benzyl L-glutamate)/Deuterated Dimenthylformamide (PBLG/d-DMF) under Shear and during Relaxation. Macromolecules, 29, 2298-2301.

https://doi.org/10.1021/ma951127p

[32] Eberle, A.P.R., Castaneda-Preigo, R., Kim, J.M. and Wagner, N.J. (2011) Dynamical Arrest, Percolation, Gelation, and Glass Formation in Model Nanoparticle Dispersions with Thermoreversible Adhesive Interactions. Langmuir, 28, 1866-1878. https://doi.org/10.1021/la2035054

[33] Teledyne Lumenera (2021) Lumenera Software Training and Tutorials. 
https://www.lumenera.com/support/industrial-usb-ethernet/training-tutorials.html

[34] Laven, P. (2012) MiePlot v4. http://www.philiplaven.com/mieplot

[35] Franck, A. (2008) Small Angle Light Scattering Option for AR Rheometers. TA Instruments Guide.

[36] TA Instruments (2008) AR Series Small Angle Light-Scattering (SALS) Accessory. TA Instruments Guide.

[37] Armstrong, M.J., Scully, M., Clark, M., Corrigan, T. and James, C. (2021) A Simple Approach for Adding Thixotropy to an Elasto-Visco-Plastic Rheological Model to Facilitate Structural Interrogation of Human Blood. Journal of Non-Newtonian Fluid Mechanics, 290, Article ID: 104503. https://doi.org/10.1016/j.jnnfm.2021.104503

[38] Armstrong, M.J., Baker, J., Trump, J., Miller, E., Wickiser, J., Cameron, K., Clark, N., Schwarting, K., Brown, T., Bailey, D., James, C., Nguyen, C. and Corrigan, T. (2021) Structure-Rheology Elucidation of Human Blood via SPP Framework and TEVP Modeling. Korea-Australia Rheology Journal, 33, 45-63.

https://doi.org/10.1007/s13367-021-0005-1

\section{Nomenclature}

\begin{tabular}{|l|l|}
\hline$\omega$ & frequency of oscillation \\
\hline$\gamma_{0}$ & strain amplitude \\
\hline $\mathrm{t}$ & time \\
\hline $\mathrm{T}$ & temperature \\
\hline$\lambda$ & structure parameter \\
\hline $\mathrm{G}$ & elastic modulus \\
\hline$\sigma_{\mathrm{y}}$ & yield stress \\
\hline $\mathrm{m}$ & power law \\
\hline$\tau_{\mathrm{C}}$ & Cross model time constant \\
\hline$\tau_{\mathrm{B}}$ & thixotropic time constant of shear breakdown \\
\hline $\mathrm{k}_{\mathrm{Break}}$ & thixotropic time constant of evolution \\
\hline $\mathrm{k}_{\text {Aggr }}$ & char. Time constant of structure aggregation \\
\hline $\mathrm{k}_{\mathrm{Brown}}$ & char. Time cosntat of Brownian motion \\
\hline$\gamma_{\mathrm{e}}$ & elasti strain \\
\hline$\gamma_{\mathrm{p}}$ & plastic strain \\
\hline$\gamma_{\text {max }}$ & maximum strain \\
\hline$\gamma_{0, \mathrm{c}}$ & critical rouleaux strain \\
\hline$\gamma$ & strain \\
\hline$\dot{\gamma}_{\mathrm{e}, \mathrm{p}}$ & elastic, plastic shear rate \\
\hline$\sigma$ & stress \\
\hline$\gamma_{\mathrm{f}}$ & shear rate \\
\hline $\mathrm{F}_{\text {cost }}$ & cost function \\
\hline$\eta \infty$ & infinite shear viscosity \\
\hline $\mathrm{d}$ & power law of shear aggr. \\
\hline $\mathrm{a}$ & power law of shear breakdown \\
\hline & \\
\hline
\end{tabular}

